\title{
Demagnetizing factors for two parallel ferromagnetic plates and their applications to
} magnetoelectric laminated sensors

E. Liverts, A. Grosz, B. Zadov, M. I. Bichurin, Y. J. Pukinskiy, S. Priya, D. Viehland, and E. Paperno

Citation: Journal of Applied Physics 109, $07 D 703$ (2011); doi: 10.1063/1.3536518

View online: http://dx.doi.org/10.1063/1.3536518

View Table of Contents: http://scitation.aip.org/content/aip/journal/jap/109/7?ver=pdfcov

Published by the AIP Publishing

\section{Articles you may be interested in}

Dynamic magnetostrictive properties of magnetization-graded ferromagnetic material and application in magnetoelectric composite

J. Appl. Phys. 115, 17C726 (2014); 10.1063/1.4866089

Converse magnetoelectric effect in ferromagnetic shape memory alloy/piezoelectric laminate

Appl. Phys. Lett. 95, 022501 (2009); 10.1063/1.3179146

Experimental evidence of end effects in magneto-electric laminate composites

J. Appl. Phys. 102, 124901 (2007); 10.1063/1.2822455

Voltage gain effect in a ring-type magnetoelectric laminate

Appl. Phys. Lett. 84, 4188 (2004); 10.1063/1.1756676

Large high-frequency magnetoelectric response in laminated composites of piezoelectric ceramics, rare-earth iron alloys and polymer

Appl. Phys. Lett. 84, 3516 (2004); 10.1063/1.1739277

MIT LINCOLN

LABORATORY CAREERS

Discover the satisfaction of innovation and service

to the nation
- Space Control

- Air \& Missile Defense

- Communications Systems \& Cyber Security

- Intelligence, Surveillance and

Reconnaissance Systems

- Advanced
Electronics
- Tactical Systems
- Homeland
Protection
- Air Traffic Control

LINCOLN LABORATORY

MassachusetTs Institute of TeChNOLOgY

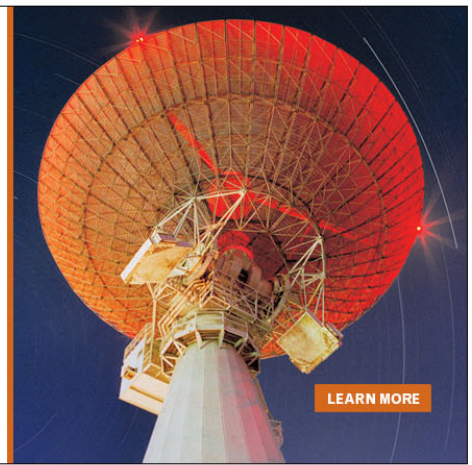




\title{
Demagnetizing factors for two parallel ferromagnetic plates and their applications to magnetoelectric laminated sensors
}

\author{
E. Liverts, ${ }^{1,2, a)}$ A. Grosz, ${ }^{2}$ B. Zadov, ${ }^{2}$ M. I. Bichurin, ${ }^{3}$ Y. J. Pukinskiy, ${ }^{3}$ S. Priya,,${ }^{4}$ D. Viehland, ${ }^{4}$ \\ and E. Paperno ${ }^{2}$ \\ ${ }^{1}$ Department of Mechanical Engineering, Ben-Gurion University of the Negev, P.O. Box 653, Beer-Sheva \\ 84105, Israel \\ ${ }^{2}$ Department of Electrical and Computer Engineering, Ben-Gurion University of the Negev, P.O. Box 653, \\ Beer-Sheva 84105, Israel \\ ${ }^{3}$ Department of Design and Technology of Radioequipment, Novgorod State University, Veliky Novgorod \\ 173003, Russia \\ ${ }^{4}$ Department of Material Science and Engineering, Virginia Tech, Blacksburg, Virginia 24061, USA
}

(Presented 15 November 2010; received 22 September 2010; accepted 25 October 2010; published online 18 March 2011)

\begin{abstract}
An analytical expression is derived to approximate the magnetometric demagnetizing factors for two parallel ferromagnetic plates having the shape of rectangular prisms. The magnetometric demagnetizing factors relate the average magnetic fields within the plates' volumes to an external magnetic field. Knowing this relationship is essential for describing the response of magnetoelectric sensors comprising two parallel magnetostrictive plates. It is shown that two separate ferromagnetic layers provide better field sensitivity than a single layer with a doubled thickness. The obtained results are in a good agreement with numerical calculations and experimental data. (C) 2011 American Institute of Physics. [doi:10.1063/1.3536518]
\end{abstract}

\section{INTRODUCTION}

Magnetostrictive-piezoelectric laminates exhibiting magnetoelectric (ME) effect have recently drawn increasing interest due to their potential for many modern devices, such as sensors, gyrators, energy harvesters, etc. ${ }^{1,2}$ A typical ME laminate comprises two parallel identical magnetostrictive plates separated by a piezoelectric layer. Its sensitivity is described by the electric-field ME coefficient, $\alpha_{E}$, relating the induced electric field to the applied magnetic field. The existing expression for $\alpha_{E}$ (Refs. 1-5) neglects the finite laminate size and its shape. However, experimental study (see, e.g., Ref. 6 and our data discussed in the following) shows that the laminate size and shape have a considerable effect on the ME coefficient.

An important factor affecting the ME coefficient is the shape demagnetization. Considering it, the reduction of the magnetic fields within the ferromagnetic layers and the corresponding reduction of $\alpha_{E}$ can be taken into account. The above-mentioned field reduction can be approximated via the magnetometric demagnetizing factor, $N^{m}, 7,8$ relating the average magnetic field within each ferromagnetic plate to the external uniform magnetic field. For two parallel ferromagnetic plates, $N^{m}$ depends on the plates' shape (being different, e.g., for prism and disk), their magnetic state (saturated or nonsaturated), and the distance between the plates.

It is a difficult task to find a general analytical solution for $N^{m}$ in the above-mentioned case. To the best of our

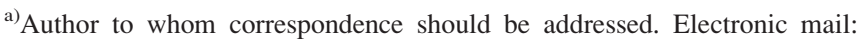
eliverts@bgu.ac.il.
}

knowledge, existing literature suggests solutions only for single prisms or cylinders. ${ }^{8,9}$

In this paper, we are bridging this gap and report on an analytical solution for $N^{m}$ for two parallel rectangular ferromagnetic prisms. We assume that the prisms' material is close to magnetic saturation, so its relative magnetic permeability $\mu_{r}$ is rather low for small ac fields. This matches the magnetization quiescent points of ME sensors in practical applications.

The found magnetic field reduction is used to correct the $\alpha_{E}$ coefficient. The obtained analytical results are compared against numerical calculations based on a finite-element method (FEM) software. The corrected $\alpha_{E}$ is compared with the measured one for a variety of ME laminate samples. Good agreement among the theory, numerical calculations, and experiment has been obtained.

\section{DEMAGNETIZING FACTORS}

To derive an expression for the magnetometric demagnetizing factors, the average magnetic field within each magnetic plate should be found. To alleviate the estimation of the average magnetic fields, we impose the following practical restrictions.

For a polycrystalline magnetostrictive ferromagnetic, pseudopiezomagnetic coefficient $q$, which is a factor in the expression for $\alpha_{E}$, (Refs. 1-5) can be estimated by

$$
q=\frac{9 \lambda}{2 M_{s}} \chi_{r} \cos \theta,
$$

where $\lambda$ is the magnetostriction coefficient averaged over the crystallographic axis of the easy magnetization, $M_{s}$ is the 
saturation magnetization, $\chi_{r}=\mu_{r}-1$ is the relative differential magnetic susceptibility, $\theta$ is the angle between the magnetization, $\mathbf{M}$, and the applied dc magnetic bias, $\mathbf{H}$. Both $\chi_{r}$ and $\theta$ depend strongly on $\mathbf{H}$. Their product, being maximal at some optimal bias, is chosen as a quiescent point for the sensor operation, see Refs. 1-5. At this point, the ferromagnetic plates are nearly saturated in terms of their magnetization, and, hence, $\chi_{r}$ is rather small. For a small ac external field $\mathbf{h}^{\text {ext }}$, that superimposes $\mathbf{H}$, the ferromagnetic material behaves like a paramagnetic one.

To reduce the demagnetization, ME sensors are elongated and directed along the $\mathbf{H}$ and $\mathbf{h}^{\text {ext }}$ fields. In such a case, the demagnetization can be estimated by employing the perturbation theory in the magnetostatics integral equation, ${ }^{10}$

$$
H_{i}(\mathbf{r})=H_{i}^{\text {ext }}+\frac{1}{4 \pi \mu_{0}} \frac{\partial^{2}}{\partial x_{i} \partial x_{k}} \int_{V} \frac{M_{k}\left(\mathbf{r}^{\prime}\right)}{\left|\mathbf{r}-\mathbf{r}^{\prime}\right|} d^{3} \mathbf{r}^{\prime} .
$$

Here, $\mathbf{r}$ is an observation point, $\mathbf{r}^{\prime}$ are the points inside the ferromagnetic plates, $V$ is the joint domain of the ferromagnetic plates, $\mu_{0}$ is the vacuum permeability, and the subscripts $i$ and $k$ denote the Cartesian components.

Iterating it once around $\mathbf{H}^{\text {ext }}=\mathbf{H}+\mathbf{h}^{\text {ext }}$, we get

$$
H_{i}(\mathbf{r}) \approx H_{i}^{\mathrm{ext}}-\frac{M\left(H_{i}^{\mathrm{ext}}\right)}{\mu_{0}} N_{i i}(\mathbf{r}),
$$

where $M\left(H_{i}^{\text {ext }}\right)$ is the magnetization curve value at an instant magnetic field $H_{i}^{\text {ext }}$ and

$$
N_{i i}(\mathbf{r})=-\frac{1}{4 \pi} \frac{\partial^{2}}{\partial x_{i}^{2}} \int_{V} \frac{d^{3} \mathbf{r}^{\prime}}{\left|\mathbf{r}-\mathbf{r}^{\prime}\right|} .
$$

In Eqs. (3) and (4) we consider only one component of tensor $N_{i k}$, because the leading term in the products of all its other components and $M\left(H_{i}^{\text {ext }}\right)$ equal zero in Eq. (3). Taking into account that, about the quiescent point, the magnitude of $\mathbf{M}$ is nearly constant over the plates' domains and averaging Eq. (3) over a single plate volume $V_{1}$, we obtain the magnetometric demagnetization factor along the $i$ axis,

$$
N_{i}^{m}=-\frac{1}{4 \pi V_{1}} \int_{V_{1}} d^{3} \mathbf{r} \frac{\partial^{2}}{\partial x_{i}^{2}} \int_{V} \frac{d^{3} \mathbf{r}^{\prime}}{\left|\mathbf{r}-\mathbf{r}^{\prime}\right|} .
$$

For two identical rectangular prisms with dimensions $l \times w \times t_{m}$, separated by a nonmagnetic layer of thickness $t_{p}$, the integration in Eq. (5) yields

$$
\begin{aligned}
N_{i}^{m}= & N_{i}^{A}\left(l, w, t_{m}\right)+\left(1+\frac{t_{p}}{2 t_{m}}\right) N_{i}^{A}\left(l, w, t_{p}+2 t_{m}\right) \\
& -\left(1+\frac{t_{p}}{t_{m}}\right) N_{i}^{A}\left(l, w, t_{p}+t_{m}\right)+\frac{t_{p}}{2 t_{m}} N_{i}^{A}\left(l, w, t_{p}\right),
\end{aligned}
$$

where $N_{i}^{A}(l, w, t)$ is given by Eq. (1) derived in Ref. 8.

\section{MAGNETIC FIELD REDUCTION}

To approximate the field reduction within the ferromagnetic plates via the magnetometric demagnetizing factors, we linearize Eq. (3) about the quiescent point $\mathbf{H}$ and average the result over the volume of one of the plates. For $\chi_{r} N_{i}^{m} \ll 1$, this gives us the averaged magnetic reduction factor that relates the $i$ component of the averaged $\mathbf{h}^{\text {int }}(\mathbf{r})$ to that of $\mathbf{h}^{\mathrm{ext}}$,

$$
\left\langle r_{i}\right\rangle=\frac{\left\langle h_{i}^{\text {int }}(\mathbf{r})\right\rangle}{h_{i}^{\text {ext }}} \approx \frac{1}{1+\chi_{r} N_{i}^{m}} .
$$

We assume that Eq. (7) can be extrapolated to a range of $\chi_{r} N_{i}^{m}>1$ because, for a single ellipsoidal plate, it gives the exact solution for any values of $\chi_{r} N_{i}^{m} \cdot{ }^{11,12}$ For example, for $i=z$, i.e., for the bias and small ac fields applied normally to the ferromagnetic plates, $N_{i}^{m} \approx 1$, according to Eq. (6), and $\left\langle r_{i}\right\rangle \approx 1 / \mu_{r}$, according to Eq. (7). Such a $\mu_{r}$-fold decrease in the internal field matches the experimental data given in Refs. 1, 4, and 5.

To compare between the analytical approximation of $\left\langle r_{i}\right\rangle$ and its numerical calculations, we have solved an example given in Table I for two ferromagnetic plates with $\mu_{\mathbf{r}}=2.5$, width $w=6 \mathrm{~mm}$, and thickness $t_{m}=0.5 \mathrm{~mm}$, separated by a piezoelectric layer with thickness $t_{p}$. The numerical values are denoted by overtildes $(\sim)$. The external magnetic field is applied along the laminate length. To find the theoretical values of $N_{i}^{m}$ and $\left\langle r_{i}\right\rangle$ in Table I, we have used Eqs. (6) and (7). To find the numerical values $\left\langle\tilde{r}_{i}\right\rangle$ of $\left\langle r_{i}\right\rangle$, we have used the COMSOL three-dimensional FEM software. $\tilde{N}_{i}^{m}$ in Table I have been obtained by solving Eq. (7) for given $\left\langle\tilde{r}_{i}\right\rangle$.

Table I demonstrates close agreement between theoretical and numerical results. This agreement is especially close (better than 98.5\%) for the reduction factors $\left\langle r_{i}\right\rangle$ because, for

\begin{tabular}{|c|c|c|c|c|c|c|c|c|c|c|c|c|c|c|c|c|}
\hline \multirow[b]{2}{*}{$l(\mathrm{~mm})$} & \multicolumn{4}{|c|}{$t_{p}=0.25 \mathrm{~mm}$} & \multicolumn{4}{|c|}{$t_{p}=1.0 \mathrm{~mm}$} & \multicolumn{4}{|c|}{$t_{p}=2.0 \mathrm{~mm}$} & \multicolumn{4}{|c|}{$t_{p}=4.0 \mathrm{~mm}$} \\
\hline & $N_{i}^{m}$ & $\tilde{N}_{i}^{m}$ & $\left\langle r_{i}\right\rangle$ & $\left\langle\tilde{r}_{i}\right\rangle$ & $N_{i}^{m}$ & $\tilde{N}_{i}^{m}$ & $\left\langle r_{i}\right\rangle$ & $\left\langle\tilde{r}_{i}\right\rangle$ & $N_{i}^{m}$ & $\tilde{N}_{i}^{m}$ & $\left\langle r_{i}\right\rangle$ & $\left\langle\tilde{r}_{i}\right\rangle$ & $N_{i}^{m}$ & $\tilde{N}_{i}^{m}$ & $\left\langle r_{i}\right\rangle$ & $\left\langle\tilde{r}_{i}\right\rangle$ \\
\hline 5.0 & 0.145 & 0.133 & 0.822 & 0.833 & 0.126 & 0.116 & 0.841 & 0.852 & 0.115 & 0.105 & 0.853 & 0.864 & 0.096 & 0.107 & 0.863 & 0.874 \\
\hline 10.0 & 0.081 & 0.072 & 0.892 & 0.903 & 0.071 & 0.064 & 0.904 & 0.913 & 0.065 & 0.058 & 0.911 & 0.920 & 0.053 & 0.060 & 0.918 & 0.926 \\
\hline 15.0 & 0.056 & 0.049 & 0.923 & 0.932 & 0.049 & 0.044 & 0.931 & 0.949 & 0.045 & 0.040 & 0.937 & 0.944 & 0.042 & 0.037 & 0.941 & 0.948 \\
\hline 20.0 & 0.043 & 0.037 & 0.940 & 0.948 & 0.038 & 0.033 & 0.947 & 0.953 & 0.035 & 0.030 & 0.951 & 0.957 & 0.032 & 0.028 & 0.954 & 0.960 \\
\hline 25.0 & 0.034 & 0.030 & 0.951 & 0.958 & 0.030 & 0.026 & 0.956 & 0.962 & 0.028 & 0.024 & 0.960 & 0.965 & 0.026 & 0.022 & 0.963 & 0.968 \\
\hline 30.0 & 0.029 & 0.025 & 0.959 & 0.965 & 0.026 & 0.022 & 0.963 & 0.968 & 0.024 & 0.020 & 0.966 & 0.971 & 0.022 & 0.019 & 0.968 & 0.973 \\
\hline
\end{tabular}
$\chi_{r} N_{i}^{m} \ll 1$, which is the case of Table I, the sensitivity of $\left\langle r_{i}\right\rangle$ to $N_{i}^{m}$ is very low.

To verify our theory experimentally, we compare in Fig. 1 the theoretical and experimental values of the ME coefficient $\alpha_{E, 31}$. The theoretical values of $\alpha_{E, 31}$ were obtained as a

TABLE I. Theoretical and numerical values of the magnetometric demagnetizing and reduction factors, $N_{i}^{m}$ and $\left\langle r_{i}\right\rangle$. 


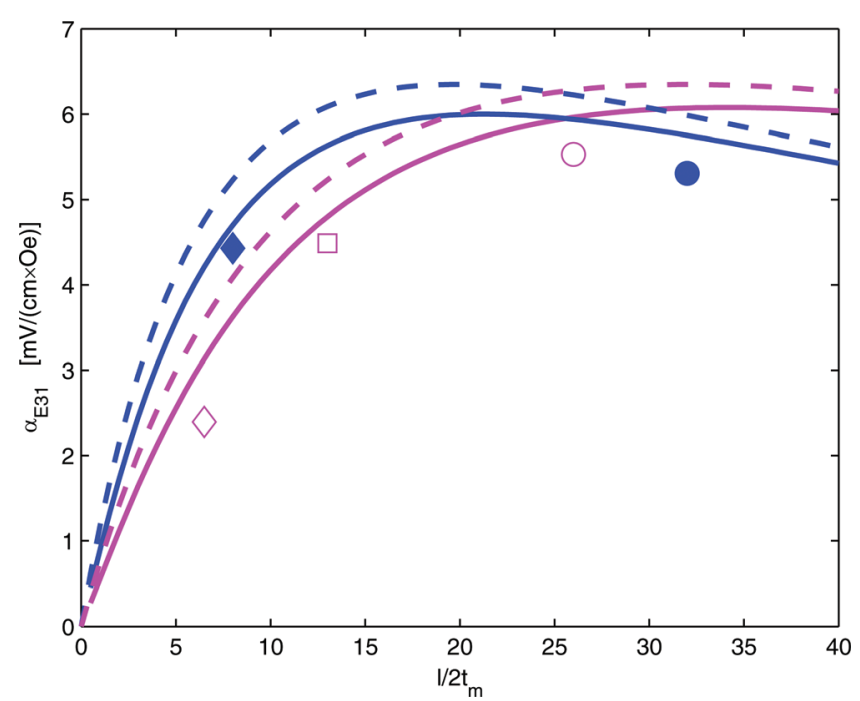

FIG. 1. (Color online) The ME coefficient $\alpha_{E 31}$ calculated without (dashed lines) and with (solid lines) the correction due to the shape demagnetization. The markers show experimental data for the samples with: $t_{p}=0.5 \mathrm{~mm}$, $13 \times 6 \times 0.25 \mathrm{~mm}$ (open circle), $13 \times 6 \times 0.5 \mathrm{~mm}$ (open square), $13 \times 6 \times 1$ $\mathrm{mm}$ (open diamond), $t_{p}=1.0 \mathrm{~mm}, 16 \times 6 \times 0.25 \mathrm{~mm}$ (closed circle), $16 \times 6 \times 1 \mathrm{~mm}$ (closed diamond).

function of $l / 2 t_{m}$ by using the standard expression given in Refs. 1, 4, and 5 for the external magnetic field applied along the laminate length. To consider the shape demagnetization, the obtained $\alpha_{E, 31}$ was multiplied by the reduction factor $\left\langle r_{i}\right\rangle$. The following parameters were used in theoretical calculations: the piezomagnetic modulus $q_{31}=4.7 \times 10^{-10} \mathrm{Oe}^{-1}$ (Ni), piezoelectric modulus $d_{31}=325 \times 10^{-12} \mathrm{C} / \mathrm{N}$, relative permittivity $\varepsilon_{r}=3400$ (APC PZT-854), Young's modulus $Y_{m}=200 \mathrm{GPa}(\mathrm{Ni}), Y_{p}=59 \mathrm{GPa}$ (APC PZT-854), and Poisson ratios $v_{m}=0.31, v_{p}=0.30$. The experimental samples were fabricated by bonding two Ni plates with an APC PZT-854 plate.

Figure 1 shows that considering the shape demagnetization improves the agreement between the theory and experiment. The improvement is a especially noticeable for $l / 2 t_{m}<40$.

\section{CONCLUSION}

An analytical expression has been obtained to describe the magnetometric demagnetizing factors $N_{i}^{m}$ for two parallel rectangular ferromagnetic prisms. This description is a natural extension of the expression for a single ferromagnetic prism. ${ }^{8}$ For $t_{p} \rightarrow 0$ or $t_{p} \rightarrow \infty$, the solution for two plates can be replaced by that for a single plate with the thickness of either $2 t_{m}$ or $t_{m}$, respectively. For these two extreme cases, our expression for $N_{i}^{m}$ becomes identical to that given in Ref. 8 .

It has been shown that, under the given conditions, $N_{i}^{m}$ and $\mu_{r}$ determine the average magnetic field inside the plates that is induced by a small applied external field. The obtained relation between the internal and external magnetic fields has been used to correct the existing expression for the ME coefficient. The corrected ME coefficient agrees well with our experimental data.

Our Eqs. (6) and (7) show that the shape demagnetization for two separate ferromagnetic plates is always weaker than that for a single plate with a double thickness. For this reason, it is better to use in ME sensors two thin magnetic layers instead of a thick one.

\section{ACKNOWLEDGMENTS}

The authors thank Dr. M. Auslender for some technical help and discussion of the results. M.I.B. and Y.J.P. thank Professor E. Paperno for warm hospitality during their stay at Ben-Gurion University where this work was partially done.

${ }^{1}$ C.-W. Nan, M. I. Bichurin, S. Dong, D. Viehland, and G. Srinivasan, J. Appl. Phys. 103, 031101 (2008).

${ }^{2}$ G. Srinivasan, Annu. Rev. Mater. Res. 40, 153 (2010).

${ }^{3}$ G. Harshe, J. P. Dougherty, and R. E. Newnham, Int. J. Appl. Electromagn. Mater. 4, 145 (1993).

${ }^{4}$ M. I. Bichurin, V. M. Petrov, and G. Srinivasan, J. Appl. Phys. 92, 7681 (2002).

${ }^{5}$ M. I. Bichurin, V. M. Petrov, and G. Srinivasan, Phys. Rev. B 68, 054402 (2003).

${ }^{6}$ D. A. Pan, J. Lu, Y. Bai, W. Y. Chu, and L. J. Qiao, Chin. Sci. Bull. 53, 2124 (2008).

${ }^{7}$ C. D. Graham and B. E. Lorenz, J. Appl. Phys. 107, 09A322 (2010).

${ }^{8}$ A. Aharoni, J. Appl. Phys. 83, 3432 (1998).

${ }^{9}$ D-X. Chen, J. A. Brug, and R. B. Goldfarb, IEEE Trans. Magn. 27, 3601 (1991).

${ }^{10}$ A. I. Akhiezer, V. G. Bar'yakhtar, and S. V. Peletminskii, Spin Waves (North-Holland, Amsterdam, 1968), p.18.

${ }^{11}$ R. M. Bozorth, Ferromagnetism (Van Nostrand, New York, 1951), p. 847.

${ }^{12}$ J. A. Osborn, Phys. Rev. 67, 351 (1945). 